\title{
Kinderdermatologie in der 3. Auflage
}

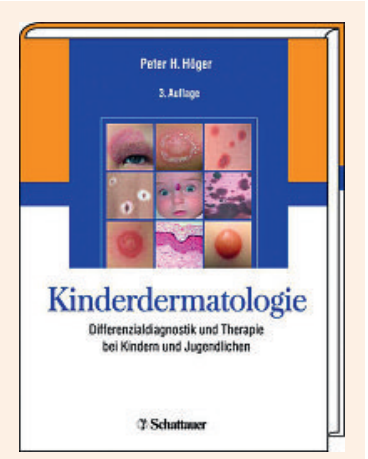

Peter H. Höger Kinderdermatologie Differenzialdiagnostik und Therapie bei Kindern und Jugendlichen 3., vollständig überarbeitete und aktualisierte Auflage Stuttgart: Schattauer; 2011 664 Seiten. 699 Abbildungen. 308 Tabellen. 179 CHF ISBN 978-3-7945-2730-4
Professor Peter Höger ist Facharzt für Pädiatrie und Dermatologie mit den Zusatzbezeichnungen Allergologie und Infektiologie und Experte auf dem Gebiet der Kinderdermatologie. Er absolvierte seine Weiterbildung in Pädiatrie an den Universitäts-Kinderkliniken Zürich, Würzburg und Hamburg. Ein Forschungsaufenthalt mit dem Schwerpunkt Pädiatrische Immunologie/Allergologie folgte in Boston, ebenso eine Weiterbildung in Dermatologie an der UniversitätsHautklinik Hamburg und in Pädiatrischer Dermatologie am Great Ormond Street Hospital for Children in London. Dass der Autor sowohl eine pädiatrische als auch eine dermatologische Ausbildung hat, kommt beiden Fachgebieten durch die Integration internistischer Aspekte zugute.

Leider kommen in der kinderärztlichen Ausbildung die Hautkrankheiten oftmals zu kurz. Den Dermatologen andererseits fehlt es oft an Erfahrung mit der Behandlung von Kindern. Daher bietet dieses vom erfahrenen Spezialisten für Pädiater und Dermatologen geschriebene Werk einen optimalen Wegweiser durch das komplexe Fachgebiet.

Auch wenn es keine grundsätzlichen Unterschiede im Aufbau der Haut gibt, unterscheidet sich jedoch die Haut eines Kindes von der eines Erwachsenen. Die kindliche Haut ist sehr viel dünner und empfindlicher und kann somit leichter geschädigt werden. Im Kindesalter treten nicht nur Ekzeme, wozu die Neurodermitis gehört, besonders häufig auf, sondern auch Schuppenflechte, Krankheiten, die mit einer Blasenbildung einhergehen, allergische Ausschläge, Infektionskrankheiten mit Beteiligung der Haut, Muttermale und einige Hauttumore.

Um Veränderungen oder Verletzungen an der Haut von Kindern und Jugendlichen sicher erkennen, einordnen und behandeln zu können, ist umfassendes dermatologisches, internistisches und pharmakologisches Wissen notwendig. Mit dem Lehr- und Nachschlagewerk von Peter Höger in der jetzt 3., aktualisierten und erweiterten Auflage ist eine Aneignung dieses Wissens gut möglich.

Übersichtlich und verständlich stellt der Autor das lebenswichtige Organ Haut vor. Prägnant weist er auf die Besonderheiten häufiger, aber auch seltener Hautkrankheiten und ihrer Therapie hin. Besonders nützlich für die tägliche Praxis sind zahlreiche Caveund Merksätze, Farbabbildungen, ausführliche Tabellen zu Differentialdiagnosen und Dosierungen sowie empfehlenswerte dermatologische Magistralrezepturen. Man findet u.a. tabellarische Übersichten kutaner Symptome systemischer Erkrankungen. Zusätzlich helfen hervorragend strukturierte Flussdiagramme, möglichst schnell von kutanen Symptomen auf das richtige Krankheitsbild zu schliessen. Diese Funktion ist auch für andere Fachärzte wertvoll, die mit Kindern und ihren Hautproblemen in Kontakt kommen, zumindest um diese mit gezielten Fragestellungen zum Spezialisten überweisen oder ggf. die Kinder und Eltern mit einer sicheren Diagnose und Therapie beruhigen zu können.

In der 3. Auflage werden als Neuerung auch Stoffwechselerkrankungen mit Hautbeteiligung, kutane Manifestationszeichen von Immundefekten sowie Hautpflege bei Neugeborenen und Säuglingen besprochen.

Die Gliederung und das Layout der einzelnen Kapitel ist ansprechend gestaltet.

Dr. med. Sandra Krüger, Berlin 